\title{
Is apolipoprotein(a) a susceptibility gene for Type I diabetes mellitus and related to long-term survival?
}

\author{
F. Kronenberg ${ }^{1}$, M. Auinger ${ }^{2}$, E. Trenkwalder ${ }^{1}$, K. Irsigler $^{2}$, G. Utermann ${ }^{1}$, H. Dieplinger ${ }^{1}$ \\ ${ }^{1}$ Institute of Medical Biology and Human Genetics, University of Innsbruck, Austria \\ ${ }^{2}$ Third Medical Department and L. Boltzmann Institute for Metabolic Diseases, Lainz Hospital, Vienna, Austria
}

\section{Abstract}

Aims/hypothesis. High lipoprotein(a) [Lp(a)] plasma concentrations are a genetically determined risk factor for atherosclerotic complications. In healthy subjects $\mathrm{Lp}(\mathrm{a})$ concentrations are mostly controlled by the apolipoprotein(a) $[\operatorname{apo}(a)]$ gene locus which determines a size polymorphism with more than 30 alleles. Subjects with low molecular weight apo (a) phenotypes on average have higher Lp(a) concentrations than those with high molecular weight apo(a) phenotypes. There are many opinions about whether and why $\operatorname{Lp}(\mathrm{a})$ is raised in patients with Type I diabetes (insulin-dependent) mellitus.

Methods. We investigated Lp(a) plasma concentrations and apo(a) phenotypes in 327 patients with Type I diabetes mellitus (disease duration 1-61 years) and in 200 control subjects matched for age and sex.

Results. Patients with a disease duration of up to 15 years had significantly higher Lp(a) concentrations $(24.3 \pm 34.0 \mathrm{mg} / \mathrm{dl}$ vs $16.7 \pm 22.6 \mathrm{mg} / \mathrm{dl}, \quad p=$ $0.014)$ compared with control subjects. This increase can be explained by a considerably higher frequency of low molecular weight apo(a) phenotypes (38.9\% vs $23.5 \%, p<0.005)$. The frequency of low molecular weight apo(a) phenotypes decreased continuously with disease duration from $41.7 \%$ in those with disease duration of up to 5 years to $18.2 \%$ in those with the disease lasting more than 35 years.

Conclusion/interpretation. Our data show that an increase of $L p(a)$ in Type I diabetic patients can only be observed in groups with short diabetes duration and that this elevation is genetically determined. Therefore, the apo $(a)$ gene, located at 6q26-27, might be a susceptibility gene for Type I diabetes mellitus which is supported by recently published studies reporting evidence for linkage of this region (6q27) with Type I diabetes mellitus. Furthermore, the decreasing frequency of low molecular weight apo $(a)$ phenotypes with disease duration suggests a survivor effect. [Diabetologia (1999) 42: 1021-1027]

Keywords Type I diabetes mellitus, apo(a) gene, apo(a) polymorphism, Lp(a), susceptibility gene, atherosclerosis, risk factor.
High lipoprotein(a) [Lp(a)] plasma concentrations have been reported to be a risk factor for coronary artery disease in most prospective studies [1]. Con-

Received: 23 December 1998 and in revised form: 8 March 1999

Corresponding author: Dr F. Kronenberg, Institute of Medical Biology and Human Genetics, Schöpfstrasse 41, A-6020 Innsbruck, Austria

Abbreviations: apo(a), apolipoprotein(a); Lp(a), lipoprotein(a); K-IV, kringle-IV; HMW, high molecular weight; LMW, low molecular weight. centrations of $\mathrm{Lp}(\mathrm{a})$ are determined strongly by the apolipoprotein(a) $[a p o(a)]$ gene locus and correlate with a size polymorphism of apo(a). The molecular basis for this size polymorphism is a varying number of kringle-IV (K-IV) copies which are highly homologous to the K-IV of plasminogen. There exists an inverse correlation between the number of K-IV repeats of apo(a) and the $\mathrm{Lp}(\mathrm{a})$ plasma concentrations. Subjects with a low number of K-IV repeats on average have high concentrations of $L p(a)$ and those with a high number of K-IV repeats show low concentrations. Investigations in the general population 
found that the apo $(a)$ gene locus at 6q26-27 co-determines the risk for coronary heart disease through its allelic control of $\mathrm{Lp}(\mathrm{a})$ plasma concentration [2-8].

There is extensive discussion whether $L p(a)$ is raised in patients with Type I diabetes mellitus $[1,9]$. Raised [10-12] and similar [12-17] concentrations compared with control subjects were reported but study groups were heterogeneous in terms of study subjects' age and duration of diabetes. Also the presence of micro- or macroalbuminuria might have influenced the results. Only few studies were originally designed to investigate whether a possible increase of $\mathrm{Lp}(\mathrm{a})$ in these patients is primarily associated with diabetes mellitus or whether this increase is secondary to the disease. These studies included apo $(a)$ phenotype analysis and came to the conclusion that apo $(a)$ is not associated with Type I diabetes mellitus $[14,17]$. Recent studies, however, reported evidence for linkage of Type I diabetes mellitus and region 6 q27 [18-22] which is within the region were the apo(a) gene is located (6q26-27).

Since diabetic patients have a tremendous risk for coronary artery disease [23-25], the group of patients under investigation has to be homogeneous in terms of age and diabetes duration so as not to diminish a possible association by a survivor effect. We therefore evaluated $\mathrm{Lp}(\mathrm{a})$ plasma concentrations and apo (a) phenotypes in 200 control subjects and 327 patients with Type I diabetes mellitus stratified by diabetes duration to address two questions. Firstly, is the apo(a) size polymorphism associated with Type I diabetes mellitus and secondly, is the $\operatorname{apo}(a)$ size polymorphism associated with long-term survival in these patients.

\section{Subjects and methods}

Patients and control subjects. We investigated 327 consecutive and unrelated Type I diabetic patients who were under observation of the outpatients department at the 3rd Medical Department of the Hospital Lainz, Vienna. The study group was recruited from the outpatient clinic during the yearly followup programme after informed consent was obtained. The study was approved by the local ethics review board. Inclusion criteria were Type I diabetes mellitus, regular attendance at the outpatient clinic in the last year and stable metabolic control with no episode of ketoacidosis in the last 3 months. Type I diabetes mellitus was defined as diabetes manifestation before the age of 30 years and insulin treatment within 1 year from diagnosis.

The 133 women and 194 men were on average $40.1 \pm$ 12.7 years old and had diabetes for $22.2 \pm 12.8$ years at the time of inclusion into the study. To compare Lp(a) plasma concentrations and apo(a) phenotype frequency, we recruited from the same geographical region a group of 200 healthy voluntary blood donors frequency matched for age and sex to the group of patients with disease duration up to 15 years.

Laboratory measurements. Patients' blood was drawn after an overnight fast. Creatinine and $\mathrm{HbA}_{1 \mathrm{c}}$ (normal value $<6 \%$ ) were analysed immediately. For other biochemical variables EDTA plasma was frozen and kept at $-80^{\circ} \mathrm{C}$ before analysis [26].

Lp(a) quantification was done as described in detail [26] with a double-antibody ELISA using an affinity-purified polyclonal apo $(a)$ antibody for coating and the horseradish peroxidase-conjugated monoclonal $1 \mathrm{~A} 2$ for detection. This antiapo(a) antibody recognises the epitope motif YYPN in kringle IV (K-IV) type 2 [27]. An Lp(a) positive serum from Immuno (Vienna, Austria) with the same apo(a) isoforms served as standard throughout the whole study. Each sample was analysed in duplicate, and intra- and interassay coefficients of variation were $2.7 \%$ and $6 \%$, respectively.

Apo (a) phenotyping was done by sodium dodecyl sulphateagarose gel electrophoresis (SDS agarose) [28] under reducing conditions, as outlined previously [29], with some minor modifications [30]. With this procedure it was possible to detect two apo (a) isoforms in $60 \%$ of the subjects under investigation. In only five subjects $(0.9 \%)$ we were not able to detect any apo(a) isoform. Apo(a) phenotyping in patients and control subjects was done at the same time and the investigators were kept unaware of the duration of diabetes mellitus.

Urinary albumin excretion was measured from an overnight sample using the turbidometric method in patients with known albustix negative urine or microalbuminuria. Patients were classified into three categories: normal albuminuria (below $20 \mu \mathrm{g} / \mathrm{min}$ ), microalbuminuria (20-200 $\mu \mathrm{g} / \mathrm{min})$ and macroalbuminuria ( $>200 \mu \mathrm{g} / \mathrm{min}$ ). In patients with macroalbuminuria or raised creatinine a 24 -h urine sample was collected to determine 24-h protein excretion. To exclude ongoing urinary infections, sediment examination was done routinely in a spontaneously voided urine sample in all patients.

Statistical analysis. Since we hypothesised an influence of diabetes duration with survival, we grouped patients in three groups according to the tertiles of diabetes duration. $\mathrm{Lp}(\mathrm{a})$ plasma concentration of patients were adjusted for $\mathrm{HbA}_{1 \mathrm{c}}$ using multivariate regression analysis. Comparisons of $\mathrm{Lp}(\mathrm{a})$ concentrations between patients (whole group and group with short diabetes duration) and control subjects were done by the nonparametric Wilcoxon rank sum test; $\mathrm{Lp}(\mathrm{a})$ concentrations between patients of different diabetes duration were compared by the Kruskal-Wallis test. We used the MantelHaenszel test for linear association to compare the frequencies of apo(a) phenotypes between diabetic patients in the tertile with long and short duration of diabetes mellitus. The frequency of LMW apo(a) phenotypes between the different groups was compared using the Pearson's $\chi^{2}$ test. We did not correct for multiple comparisons, since we had decided a priori to compare only the patient group with short diabetes duration with the control group and not the patient group with middle or long disease duration. The comparison of the whole patient group with control subjects was done additionally to show the effect of heterogeneity in terms of diabetes duration on the results.

Because of the high number of detectable apo $(a)$ isoforms ( $>30$ ), many phenotypes were only represented in low numbers (e.g. once). To account for this problem and to get sufficient sample sizes in each category, we decided a priori to combine apo (a) isoforms in steps of three K-IV repeats and to categorize phenotypes according to the molecular weight of the smaller apo(a) isoform [31]. Since phenotypes with 11-16 or more than $34 \mathrm{~K}-\mathrm{IV}$ repeats in the smaller apo $(a)$ isoform were underrepresented, we formed one group by combining 11-19 and one by combining more than $31 \mathrm{~K}-\mathrm{IV}$ repeats. In a further step, we divided apo(a) phenotypes in two subgroups according to the molecular weight of the smaller apo $(a)$ iso- 
Table 1. Lp(a) plasma concentrations and apo(a) size polymorphism in control subjects and patients with Type I diabetes mellitus. Results in patients are given separately by stratification in tertiles of diabetes duration

\begin{tabular}{|c|c|c|c|c|c|}
\hline \multirow[t]{2}{*}{ Characteristics } & \multirow{2}{*}{$\begin{array}{l}\text { Control subjects } \\
(n=200)\end{array}$} & \multirow{2}{*}{$\begin{array}{l}\text { All patients } \\
(n=327)\end{array}$} & \multicolumn{3}{|c|}{ Duration of Type I diabetes mellitus (years) } \\
\hline & & & $1-15(n=108)$ & $16-27(n=112)$ & $28-61(n=107)$ \\
\hline Age, years; mean \pm SD (median) & $29 \pm 12(28)$ & $40 \pm 13(38)$ & $31 \pm 9(29)$ & $39 \pm 9(38)$ & $51 \pm 10(52)$ \\
\hline $\mathrm{Lp}(\mathrm{a}), \mathrm{mg} / \mathrm{dl} ;$ mean $\pm \mathrm{SD}(\text { median })^{\mathrm{a}}$ & $16.7 \pm 22.6(6.8)$ & $19.1 \pm 26.1(8.6)^{\mathrm{b}}$ & $24.3 \pm 34.0(9.9)^{\mathrm{c}, \mathrm{d}}$ & $15.4 \pm 18.2(8.6)^{\mathrm{d}}$ & $17.6 \pm 22.8(6.9)^{\mathrm{d}}$ \\
\hline $\begin{array}{l}\text { Apo (a) phenotypes defined by the sm } \\
\text { 11-19 K-IV repeats } \\
\text { 20-22 K-IV repeats } \\
\text { 23-25 K-IV repeats } \\
\text { 26-28 K-IV repeats } \\
\text { 29-31 K-IV repeats } \\
\text { > 31 K-IV repeats }\end{array}$ & $\begin{array}{l}\text { ller apo(a) allele, } \\
14(7.0) \\
33(16.5) \\
30(15.0) \\
44(22.0) \\
36(18.0) \\
43(21.5)\end{array}$ & $\begin{array}{l}(\%)^{\mathrm{e}, \mathrm{f}, \mathrm{g}} \\
24(7.3) \\
66(20.2) \\
54(16.5) \\
73(22.3) \\
57(17.4) \\
53(16.2)\end{array}$ & $\begin{array}{l}12(11.1) \\
30(27.8) \\
13(12.0) \\
21(19.4) \\
14(13.0) \\
18(16.7)\end{array}$ & $\begin{array}{c}6(5.4) \\
18(16.1) \\
24(21.4) \\
27(24.1) \\
21(18.8) \\
16(14.3)\end{array}$ & $\begin{array}{c}6(5.6) \\
18(16.8) \\
17(15.9) \\
25(23.4) \\
22(20.6) \\
19(17.8)\end{array}$ \\
\hline $\begin{array}{l}\text { Apo }(a) \text { phenotype groups } \\
\text { LMW }^{\mathrm{k}} \text { apo }(a) \text { phenotypes, } n,(\%) \\
\mathrm{HMW}^{\mathrm{l}} \text { apo }(a) \text { phenotypes, } n,(\%)\end{array}$ & $\begin{array}{r}47(23.5) \\
153(76.5)\end{array}$ & $\begin{array}{r}90(27.5)^{\mathrm{h}} \\
237(72.5)\end{array}$ & $\begin{array}{l}42(38.9)^{\mathrm{i}} \\
66(61.1)\end{array}$ & $\begin{array}{l}24(21.4) \\
88(78.6)\end{array}$ & $\begin{array}{l}24(22.4)^{j} \\
83(77.6)\end{array}$ \\
\hline
\end{tabular}

${ }^{a} \operatorname{Lp}(\mathrm{a})$ plasma concentrations in patients are adjusted for $\mathrm{HbA}_{1 \mathrm{c}}$.

${ }^{\mathrm{b}} p=0.22$ by Wilcoxon rank sum test for comparison of $\mathrm{Lp}(\mathrm{a})$ plasma concentrations between control subjects and all patients.

${ }^{c} p=0.014$ by Wilcoxon rank sum test for comparison of $\mathrm{Lp}(\mathrm{a})$ plasma concentrations between patients with short disease duration (1-15 years) and control subjects.

${ }^{\mathrm{d}} p=0.17$ by Kruskal-Wallis-test for comparison of Lp(a) plasma concentrations of the three patient groups with different diabetes duration.

e Pearson's $\chi^{2}$ test comparing the frequencies of apo(a) phenotypes of all Type I diabetic patients with control subjects: $\chi^{2}=3.03, d f=5, p=0.70$.

${ }^{\mathrm{f}}$ Mantel-Haenszel test for linear association comparing the frequencies of apo(a) phenotypes between Type I diabetic patients with short disease duration (1-15 years) and control subjects: $\chi^{2}=5.8, d f=1, p=0.016$.

forms, as done in all of our and some of others previous works $[3,4,8,30,32,33]$. The low molecular weight group (LMW) included all subjects with at least one apo $(a)$ isoform with 11 to $22 \mathrm{~K}-\mathrm{IV}$ repeats [29]; the high molecular weight (HMW) group comprised all subjects who had only isoforms with more than $22 \mathrm{~K}-\mathrm{IV}$ repeats. In case two apo $(a)$ isoforms were detectable, we used only the smaller apo(a) isoform for categorization [3, $4,8,30,32,33]$.

Statistical analysis was done with Statistical Package for the Social Sciences (SPSS) for Windows 7.5.2.

\section{Results}

Comparison of Type I diabetic patients and control subjects. First, we compared Lp(a) plasma concentrations and the apo(a) phenotype distribution between the whole group of diabetic patients and the control subjects. Lp(a) was slightly raised in patients when compared with control subjects without reaching sig-
${ }^{\mathrm{g}}$ Mantel-Haenszel test for linear association comparing the frequencies of apo (a) phenotypes between Type I diabetic patients with long (28-61 years) and short (1-15 years) disease duration: $\chi^{2}=4.1, d f=1, p=0.042$.

${ }^{\mathrm{h}}$ Pearson's $\chi^{2}$ test comparing the frequencies of LMW apo(a) phenotypes of Type I diabetic patients with control subjects: $\chi^{2}=1.04, d f=1, p=0.31$.

${ }^{i}$ Pearson's $\chi^{2}$ test comparing the frequencies of LMW apo(a) phenotypes of Type I diabetic patients with short disease duration $\left(1-15\right.$ years) with control subjects: $\chi^{2}=8.08, d f=1$, $p=0.004$.

j Pearson's $\chi^{2}$ test comparing the frequencies of LMW apo(a) phenotypes between patients with long (28-61 years) and short (1-15 years) disease duration: $\chi^{2}=6.84, d f=1, p=0.009$.

$\mathrm{k}$ including all subjects with at least one apo(a) isoform with 11 to $22 \mathrm{~K}-\mathrm{IV}$ repeats.

${ }^{1}$ all subjects who had only isoforms with more than $22 \mathrm{~K}-\mathrm{IV}$ repeats.

nificance $\quad(19.1 \pm 26.1 \mathrm{mg} / \mathrm{dl} \quad$ vs $16.7 \pm 22.6 \mathrm{mg} / \mathrm{dl}$, $p=0.22$ ). The apo(a) phenotype frequency was also not significantly different in terms of number of $\mathrm{K}$ IV repeats or in terms of LMW apo(a) phenotypes (Table 1).

Since the patient group showed a wide range in the duration of diabetes mellitus from 1 to 61 years, we stratified the group in tertiles according to disease duration (1-15 years, $16-27$ years and $>27$ years). We compared the frequency of the apo(a) alleles of the group with a short duration of diabetes (1-15 years) with the control group to see whether Type I diabetes mellitus is associated with the apo(a) size polymorphism. Both groups were comparable for sex $(58.2 \%$ vs $56.0 \%$ men) and age $(30.7 \pm 9.2$ years vs $29.1 \pm 12.0)$. $\mathrm{Lp}(\mathrm{a})$ was higher in patients with short diabetes duration compared with control subjects $(24.3 \pm 34.0 \mathrm{mg} / \mathrm{dl}$ vs $16.7 \pm 22.6 \mathrm{mg} / \mathrm{dl}, \quad p=0.014)$. We also observed a pronounced difference in the 

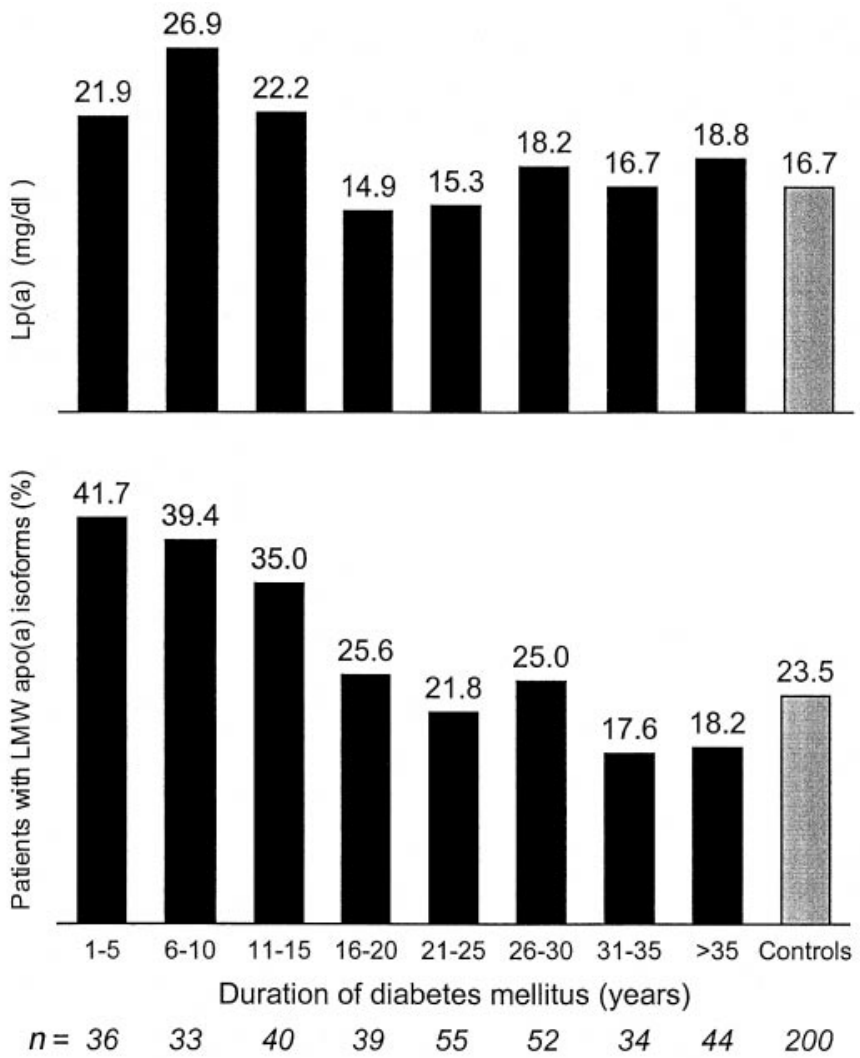

Fig.1. Lp(a) plasma concentrations and frequency of LMW apo $(a)$ isoforms in Type I diabetic patients in relation to disease duration. The frequency of LMW apo $(a)$ isoforms clearly decreased with duration of diabetes mellitus $(p=0.001$, Mantel-Haenszel test for linear association). $n$ indicates the number of subjects in each group. Results are also given for control subjects (grey bars)

apo(a) allele frequency which was caused by a preponderance of LMW apo(a) isoforms in patients with the short diabetes duration $(38.9 \%$ vs $23.5 \%$ in control subjects, $p=0.004$ ) (Table 1). Statistical calculations showed that we had $77 \%$ power at a $95 \%$ confidence level to detect this difference which is only $3 \%$ below the conventional level.

Relation to diabetes duration. We then investigated a possible survivor effect in relation to the $\mathrm{Lp}(\mathrm{a})$ plasma concentrations and the $a p o(a)$ size polymorphism. The $\operatorname{Lp}(\mathrm{a})$ concentrations tended to be lower in groups with longer duration of diabetes mellitus (Fig.1). This trend, however, did not reach significance probably due to a distinctively higher frequency of microalbuminuria and proteinuria in patients with long diabetes duration (Table 1). The apo(a) size polymorphism in terms of LMW versus HMW apo(a) phenotypes showed a clearly decreasing frequency of LMW apo $(a)$ phenotypes with the duration of diabetes mellitus $(p=0.001$, Mantel-Haenszel test for linear association). This frequency decreased from $41.7 \%$ in those with $1-5$ years disease duration to $18.2 \%$ in those with more than 35 years of disease duration (Fig.1). The disease duration was significantly shorter in patients with LMW apo(a) phenotypes compared with those with HMW phenotypes (18 \pm 12 years vs $24 \pm 12$ years, $p<0.001)$.

To test this relationship in more detail, we compared the apo(a) phenotype frequencies between patients in the tertiles with short (1-15 years) and long duration ( $>27$ years) of diabetes. The frequency of LMW apo(a) phenotypes was considerably lower in patients with long compared with those with short diabetes duration $(22.4 \%$ vs $38.9 \%, p=0.009)$ which indicates a disadvantage in long-term survival for patients with LMW apo(a) isoforms (Table 1).

To exclude that the decreasing frequency of LMW apo (a) isoforms with diabetes duration is simply an effect of age, we calculated the frequency of LMW apo(a) isoforms in the different age groups of our and of a previous control group [33]. In both control groups the frequency of LMW apo(a) isoforms did not change with age.

\section{Discussion}

Siblings of subjects with Type I diabetes mellitus have an about 15 times higher risk for diabetes mellitus than random subjects. Although the major histocompatibility complex (MHC) was found to be the major susceptibility locus for Type I diabetes mellitus, explaining $34 \%$ of the familial clustering of the disease [34], several other susceptibility loci scattered throughout the genome were described [35]. One of these loci called IDDM 8 is assigned to the chromosomal region $6 \mathrm{q} 27$ [18-22] which is within the region where the apo(a) gene is located (6q26-27) [36-38]. Therefore, apo (a) represents a very likely candidate gene for Type I diabetes mellitus.

We report for the first time that the apo(a) size polymorphism might be directly associated with Type I diabetes mellitus. We observed a noticeably higher frequency of patients with short duration of diabetes and LMW apo(a) isoforms when compared with control subjects frequency matched for sex and age. This preferential association of LMW apo $(a)$ isoforms with diabetes indicates that the apo $(a)$ gene could actually be a susceptibility gene for Type I diabetes mellitus. The alternative explanation is a linkage disequilibrium with a susceptibility locus for Type I diabetes mellitus near the apo(a) gene locus on chromosome 6q26-27 [36].

We were able to find this difference in apo(a) allele frequency only when we investigated patients stratified in groups according to diabetes duration. This might be of considerable importance since $\mathrm{Lp}$ (a) plasma concentrations and the apo(a) size polymorphism are possibly related to survival due to the association with atherosclerotic complications. 
We observed a pronounced and nearly linear decrease of the frequency of LMW apo $(a)$ phenotypes with diabetes duration (Fig. 1). Patients with a disease duration up to 5 years showed an LMW apo(a) phenotype frequency of $41.2 \%$ in comparison with only $18.2 \%$ in patients with more than 35 years of disease. It is well documented by several studies of the general population that the apo $(a)$ gene locus determines the risk for coronary heart disease through its control of $L p(a)$ plasma concentration [2-8]. A recent study described a pronounced association between LMW apo(a) phenotypes and coronary heart disease. This association was strongest for patients with coronary heart disease with younger age at onset [39]. We showed in a prevalence study including 607 haemodialysis patients [30] and in a prospective study in 440 haemodialysis patients [40] that especially apo(a) isoforms of low molecular weight and the presence of diabetes mellitus are associated with an increased risk of major cardiovascular events. There is the possibility of a negative impact of LMW apo(a) phenotypes on the survival of patients with Type I diabetes mellitus, aged about 35 to 40 years and older. It is in accordance with the decreased frequency of LMW apo(a) phenotypes we observed after a disease duration of about 20 years and more and the observation that the mortality rate due to coronary heart disease rapidly increases after age 30 years [23-25]. Lp(a) plasma concentrations did not show such a pronounced decrease with disease duration as one would expect from the decreasing frequency of LMW apo(a) phenotypes. This might be explained by the increasing microalbuminuria and proteinuria with disease duration which were shown to increase $\mathrm{Lp}(\mathrm{a})$ concentrations [13, 41-44].

Two large studies that investigated $\mathrm{Lp}(\mathrm{a})$ in diabetic children found $\mathrm{Lp}(\mathrm{a})$ concentrations to be significantly raised in patients compared with control children $[10,11]$ both of similar age or puberty stage. Studies in adult Type I diabetic patients described mostly unchanged Lp(a) concentrations [13-17]. The young age and short duration of diabetes in children might be the reason that a clear increase of $L p(a)$ was still detectable, because a cardiovascular survival effect at this age is negligible. Adult patients in most of the above studies were on average diabetic for a longer time than our group with the short diabetes duration of 1 to 15 years (average $8.2 \pm 4.4$ years). These patients would not be expected to differ much from control subjects if a survivor effect is present which decreases the prevalence of LMW apo $(a)$ isoforms with time. Therefore, studies including older patients with longer duration of diabetes might have missed an association of $\mathrm{Lp}(\mathrm{a})$ plasma concentrations or apo (a) isoforms with diabetes mellitus or both. A further argument against an increase of $\mathrm{Lp}(\mathrm{a})$ concentrations in Type I diabetic patients was a study of identical twins which found very similar $\operatorname{Lp}(\mathrm{a})$ concentrations in dia- betic patients and their healthy co-twins [45]. This observation, however, rules out diabetes as a secondary but not primary cause of high $\mathrm{Lp}(\mathrm{a})$ in Type I diabetic patients. Since identical twins share apo(a) alleles identical by descent, a genetic contribution to the increase of $L p(a)$ in Type I diabetic patients cannot be detected by such a study design.

A recent in vitro investigation observed that insulin suppresses the apo(a) synthesis in primary cultures of cynomolgus monkey hepatocytes [46]. This is in line with some studies describing a relation between poor glycaemic control in Type I diabetic patients and high $\mathrm{Lp}(\mathrm{a})$ plasma concentrations $[12,47$, 48]. We can rule out, however, that the increase of $\mathrm{Lp}(\mathrm{a})$ is secondarily caused by the changed hormonal status in our patients, since apo(a) phenotyping clearly showed a higher frequency of LMW apo(a) phenotypes in patients with short disease duration. We furthermore adjusted $\mathrm{Lp}(\mathrm{a})$ for $\mathrm{HbA}_{1 \mathrm{c}}$ values and could therefore exclude that, besides this primary effect, a secondary effect due to glycaemic control has influenced our results.

This study is a cross-sectional study with the possibility of bias. To avoid a selection bias we included consecutive and unrelated patients. Nevertheless, it is conceivable that more patients with atherosclerotic complications were attending the routine check-ups at the outpatient department. If that were the case it would increase the frequency of patients with LMW apo(a) phenotypes especially in the group with higher age and longer diabetes duration. Therefore, a dilution of the observed survivor effect for patients with HMW apo $(a)$ isoforms would be the consequence. We would not expect that atherosclerotic complications are a major reason for younger patients with a short disease duration to visit the outpatient department.

Our analysis is based on apo(a) phenotypes derived from plasma proteins and not from genomic analysis. We do not think, however, that this has influenced our findings since apo (a) phenotyping derived from plasma and from genomic DNA show a high correspondence [29]. This is especially true for LMW apo (a) isoforms which are usually accompanied with high $\mathrm{Lp}$ (a) concentrations and a high detection probability in SDS agarose gel electrophoresis.

In summary, these results suggest that LMW apo (a) phenotypes are associated with Type I diabetes mellitus and further that these isoforms are associated with a decreased probability of survival in patients with a long duration of diabetes mellitus.

Acknowledgements. We wish to thank Dr. S. C. Hunt (Cardiovascular Genetics, University of Utah, Salt Lake City) for helpful comments and critical reading of the manuscript. We thank Dr. C. Feinböck and the "Blutbank, Blutspendedienst" of the Red Cross Vienna for providing samples of healthy blood donors.

F. Kronenberg is supported by the "Austrian Programme for Advanced Research and Technology" (APART) of the 
Austrian Academy of Science. This study was supported by grants from the "Austrian Nationalbank" to F. Kronenberg (Project 5553) and H. Dieplinger (Project 6721) as well as from the Austrian "Fonds zur Förderung der wissenschaftlichen Forschung" to H. Dieplinger (P-12358).

Parts of this manuscript were presented at the 34th Annual Meeting of the European Association of the Study of Diabetes (EASD) in Barcelona, Spain, 1998.

\section{References}

1. Kronenberg F, Steinmetz A, Kostner GM, Dieplinger H (1996) Lipoprotein(a) in health and disease. Crit Rev Clin Lab Sci 33: 495-543

2. Sandholzer C, Boerwinkle E, Saha N, Tong MC, Utermann G (1992) Apolipoprotein(a) phenotypes, Lp(a) concentration and plasma lipid levels in relation to coronary heart disease in a Chinese population: evidence for the role of the $\operatorname{apo}(a)$ gene in coronary heart disease. J Clin Invest 89: 1040-1046

3. Sandholzer C, Saha N, Kark JD et al. (1992) Apo(a) isoforms predict risk for coronary heart disease: a study in six populations. Arterioscler Thromb 12: 1214-1226

4. Wild SH, Fortmann SP, Marcovina SM (1997) A prospective case-control study of lipoprotein(a) levels and apo(a) size and risk of coronary heart disease in Stanford FiveCity Project participants. Arterioscler Thromb Vasc Biol 17: 239-245

5. Kraft HG, Lingenhel A, Köchl S et al. (1996) Apolipoprotein(a) Kringle IV repeat number predicts risk for coronary heart disease. Arterioscler Thromb Vasc Biol 16: 713-719

6. Lemming L, Klausen IC, Hansen PS, Gerdes LU, Faergeman O (1995) Apolipoprotein(a) phenotypes in Danes with and without coronary heart disease. Nutr Metab Cardiovasc Dis 5: 231-236

7. Demeester CA, Bu X, Gray RJ, Lusis AJ, Rotter JI (1995) Genetic variation in lipoprotein (a) levels in families enriched for coronary artery disease is determined almost entirely by the apolipoprotein (a) gene locus. Am J Hum Genet 56: 287-293

8. Klausen IC, Sjol A, Hansen PS et al. (1997) Apolipoprotein (a) isoforms and coronary heart disease in men - a nested case-control study. Atherosclerosis 132: 77-84

9. Haffner SM (1993) Lipoprotein(a) and diabetes. Diabetes Care 16: 835-840

10. Salzer B, Stavljenic A, Jürgens G, Dumic M, Radica A (1993) Polymorphism of apolipoprotein E, lipoprotein(a), and other lipoproteins in children with type I diabetes. Clin Chem 39: 1427-1432

11. Couper JJ, Bates DJ, Cocciolone R et al. (1993) Association of lipoprotein(a) with puberty in IDDM. Diabetes Care 16: 869-873

12. Purnell JQ, Marcovina SM, Hokanson JE et al. (1995) Levels of lipoprotein(a), apolipoprotein B, and lipoprotein cholesterol distribution in IDDM - results from follow-up in the diabetes control and complications trial. Diabetes 44: $1218-1226$

13. Jenkins AJ, Steele JS, Janus ED, Best JD (1991) Increased plasma apolipoprotein(a) levels in IDDM patients with microalbuminuria. Diabetes 40: 787-790

14. Klausen IC, Berg Schmidt E, Lervang HH, Gerdes LU, Ditzel J, Faergeman O (1992) Normal lipoprotein(a) concentrations and apolipoprotein(a) isoforms in patients with insulin-dependent diabetes mellitus. Eur J Clin Invest 22: 538-541
15. Gall M-A, Rossing P, Hommel E et al. (1992) Apolipoprotein(a) in insulin-dependent diabetic patients with and without diabetic nephropathy. Scand J Clin Lab Invest 52: 513-521

16. Heller FR, Jamart J, Honore P et al. (1993) Serum lipoprotein(a) in patients with diabetes mellitus. Diabetes Care 16: 819-823

17. Császár A, Dieplinger H, Sandholzer C et al. (1993) Plasma lipoprotein(a) concentration and phenotypes in diabetes mellitus. Diabetologia 36: 47-51

18. Davies JL, Kawaguchi Y, Bennett ST et al. (1994) A genome-wide search for human type 1 diabetes susceptibility genes. Nature 371: 130-136

19. Luo DF, Bui MM, Muir A, Maclaren NK, Thomson G, She JX (1995) Affected-sib-pair mapping of a novel susceptibility gene to insulin-dependent diabetes mellitus (IDDM 8) on chromosome 6q25- q27. Am J Hum Genet 57: 911-919

20. Luo DF, Buzzetti R, Rotter JI et al. (1996) Confirmation of three susceptibility genes to insulin-dependent diabetes mellitus: IDDM4, IDDM5 and IDDM8. Hum Mol Genet 5: 693-698

21. Davies JL, Cucca F, Goy JV et al. (1996) Saturation multipoint linkage mapping of chromosome $6 \mathrm{q}$ in type 1 diabetes. Hum Mol Genet 5: 1071-1074

22. Gorus FK, Belgian Diabetes Registry (1997) Diabetes registries and early biological markers of insulin-dependent diabetes mellitus. Diabetes Metab Rev 13: 247-274

23. Krolewski AS, Kosinski EI, Warram JH et al. (1987) Magnitude and determinants of coronary artery disease in juvenile-onset, insulin-dependent diabetes mellitus. Am J Cardiol 59: 750-755

24. Portuese EI, Kuller L, Becker D, Ellis D, Lloyd CE, Orchard TJ (1995) High mortality from unidentified CVD in IDDM: time to start screening? Diabetes Res Clin Pract 30: 223-231

25. Jensen T, Borch-Johnsen K, Kofoed-Enevoldsen A, Deckert $\mathrm{T}$ (1987) Coronary heart disease in young type 1 (insulin-dependent) diabetic patients with and without diabetic nephropathy: incidence and risk factors. Diabetologia 30: 144-148

26. Kronenberg F, Lobentanz E-M, König P, Utermann G, Dieplinger H (1994) Effect of sample storage on the measurement of lipoprotein(a), apolipoproteins B and A-IV, total and high-density lipoprotein cholesterol and triglycerides. J Lipid Res 35: 1318-1328

27. Dieplinger H, Gruber G, Krasznai K et al. (1995) Kringle 4 of human apolipoprotein[a] shares a linear antigenic site with human catalase. J Lipid Res 36: 813-822

28. Kamboh MI, Ferrell RE, Kottke BA (1991) Expressed hypervariable polymorphism of apolipoprotein(a). Am J Hum Genet 49: 1063-1074

29. Kraft HG, Lingenhel A, Bader G, Kostner GM, Utermann G (1996) The relative electrophoretic mobility of apo(a) isoforms depends on the gel system: proposal of a nomenclature for apo(a) phenotypes. Atherosclerosis 125: 53-61

30. Koch M, Kutkuhn B, Trenkwalder E et al. (1997) Apolipoprotein B, fibrinogen, HDL cholesterol and apo(a) phenotypes predict coronary artery disease in hemodialysis patients. J Am Soc Nephrol 8: 1889-1898

31. Budowle B, Giusti AM, Waye JS et al. (1991) Fixed-bin analysis for statistical evaluation of continuous distributions of allelic data from VNTR loci, for use in forensic comparisons. Am J Hum Genet 48: 841-855

32. Kronenberg F, Kathrein H, König P et al. (1994) Apolipoprotein(a) phenotypes predict the risk for carotid atherosclerosis in patients with end-stage renal disease. Arterioscler Thromb 14: 1405-1411 
33. Kronenberg F, König P, Neyer U et al. (1995) Multicenter study of lipoprotein(a) and apolipoprotein(a) phenotypes in patients with end-stage renal disease treated by hemodialysis or continuous ambulatory peritoneal dialysis. J Am Soc Nephrol 6: 110-120

34. Mein CA, Esposito L, Dunn MG et al. (1998) A search for type 1 diabetes susceptibility genes in families from the United Kingdom. Nat Genet 19: 297-300

35. Todd JA, Farrall M (1996) Panning for gold: genome-wide scanning for linkage in type 1 diabetes. Hum Mol Genet 5: 1443-1448

36. Murray JC, Buetow KH, Donovan M et al. (1987) Linkage disequilibrium of plasminogen polymorphisms and assignment of the gene to human chromosome 6q26-6q27. Am J Hum Genet 40: 338-350

37. Frank SL, Klisak I, Sparkes RS et al. (1988) The apolipoprotein (a) gene resides on human chromosome 6q26-27 in close proximity to the homologous gene for plasminogen. Hum Genet 79: 352-356

38. Lindahl G, Gersdorf E, Menzel HJ et al. (1989) The gene for the $\mathrm{Lp}(\mathrm{a})$-specific glycoprotein is closely linked to the gene for plasminogen on chromosome 6 . Hum Genet 81: $149-152$

39. Gazzaruso C, Garzaniti A, Buscaglia P et al. (1999) Association between apolipoprotein(a) phenotypes and coronary heart disease at a young age. J Am Coll Cardiol 33: 157-163

40. Kronenberg F, Neyer U, Lhotta K et al. (1999) The low molecular weight apo(a) phenotype is an independent risk fac- tor for coronary artery disease in hemodialysis patients: a prospective follow-up. J Am Soc Nephrol 10: 1027-1036

41. Kapelrud H, Bangstad H-J, Dahl-Jorgensen K, Berg K, Hanssen KF (1991) Serum Lp(a) lipoprotein concentrations in insulin dependent diabetic patients with microalbuminuria. BMJ 303: 675-678

42. Gazzaruso C, Garzaniti A, Buscaglia P et al. (1998) Lipoprotein(a) levels and apolipoprotein(a) polymorphism in type 1 diabetes mellitus: relationships to microvascular and neurological complications. Acta Diabetol 35: 13-18

43. Karádi I, Romics L, Pálos G et al. (1989) Lp(a) lipoprotein concentration in serum of patients with heavy proteinuria of different origin. Clin Chem 35: 2121-2123

44. Kronenberg F, Utermann G, Dieplinger H (1996) Lipoprotein(a) in renal disease. Am J Kidney Dis 27: 1-25

45. Dubrey SW, Reaveley DR, Seed M et al. (1994) Risk factors for cardiovascular disease in IDDM - a study of identical twins. Diabetes 43: 831-835

46. Neele DM, de Wit EC, Princen HM (1999) Insulin suppresses apolipoprotein(a) synthesis by primary cultures of cynomolgus monkey hepatocytes. Diabetologia 42: 41-44

47. Haffner SM, Tuttle KR, Rainwater DL (1991) Decrease of lipoprotein(a) with improved glycemic control in IDDM subjects. Diabetes Care 14: 302-307

48. Ramirez LC, Arauz-Pacheco C, Lackner C, Albright G, Adams BV, Raskin P (1992) Lipoprotein (a) levels in diabetes mellitus: relationship to metabolic control. Ann Intern Med 117: 42-47 